\title{
Modelagem da População de Jacaré Negro dos Esteros de Ibera ${ }^{1}$
}

M.L. de CASTRO ${ }^{2}$, J.A.L. da SILVA, Departamento de Matemática Pura e Aplicada, Universidade Federal do Rio Grande do Sul, Av. Bento Gonçalves, 9500, Porto Alegre, RS, Brasil.

\begin{abstract}
Resumo. Um modelo não-linear de estrutura etária foi construído para estudar a dinâmica da população de C. yacare dos Esteros de Ibera, ao norte da Argentina. Nosso modelo considera a influência do nível da água do pântano na taxa de sobrevivência da população. A localização dos ninhos (que está diretamente relacionada com a temperatura dos ninhos) é considerada como o fator determinante para o sexo dos recém nascidos. O comportamento dinâmico da população é observado através de um programa de simulação numérica, desenvolvido especialmente para este fim, que inclui opções para o estudo de viabilidade de estratégias de desenvolvimento, como coleta de ovos e caça.
\end{abstract}

\section{Introdução}

A pressão para a exploração econômica em reservas ambientais cresce a cada dia, fazendo-se necessário a avaliação do impacto ambiental que tal estratégia de desenvolvimento pode vir a causar. O projeto The sustainable management of wetland resources in Mercosur tem por objetivo estudar a viabilidade do desenvolvimento sustentável nos Esteros de Ibera, através da cooperação de cerca de dez universidades, entre elas a Universidade Federal do Rio Grande do Sul, encarregada da modelagem matemática da população do jacaré negro, uma das espécies chave de valor comercial da região.

Projetos semelhantes em Louisiana e Botswana sobre o American Alligator foram realizados nos anos 70, compondo nossa principal referência bibliográfica ([1],[3]). Estes trabalhos nos serviram de base para modelar a taxa de sobrevivência, que é composta por uma série de mortalidades por causas específicas, como predação, canibalismo e desidratação; além de enriquecer nosso conhecimento sobre os aspectos biológicos da espécie. Além destes, o modelo proposto por Woodward e Murray [6] serviu de base para nosso modelo, que considera a localização do ninho, por estar diretamente ligada a temperatura de incubação dos ovos, como fator determinante para a razão entre machos e fêmeas no ninho.

\footnotetext{
${ }^{1}$ Inteiramente financiado pela Comunidade Européia (contrato número ERBIC 18-CT98-0262)

${ }^{2}$ manuela@mat.ufrgs.br
} 


\section{O Modelo}

O sexo dos crocodilianos é determinado pela temperatura de incubação dos ovos. Em trabalho realizado no Pantanal, Campos coletou ovos de C. yacare com temperaturas iniciais diferentes para serem incubados a $30^{\circ} \mathrm{C}$ em laboratório, determinando assim a temperatura crítica de $30.5^{\circ} \mathrm{C}$ e o periodo crítico de 40 dias para determinação do sexo. Abaixo de $30.5^{\circ} \mathrm{C}$, todos os ovos deram origem a fêmeas, exceto os que se encontravam a temperaturas superiores a $30.5^{\circ} \mathrm{C}$ e com mais de 40 dias de incubação. Para temperaturas superiores a $30.5^{\circ} \mathrm{C}$ ocorreu o nascimento de machos, em taxas diferenciadas de acordo com a vegetação do local onde os ninhos foram encontrados. Os ovos encontrados em ninhos na floresta, a uma temperatura entre $30.5^{\circ} \mathrm{C}$ e $31.5^{\circ} \mathrm{C}$ produziram $10 \%$ de machos, e a uma temperatura inicial superior a $31.5^{\circ} \mathrm{C}$ produziram de $80 \%$ a $100 \%$ de machos. Já os encontrados em mata flutuante, a temperaturas entre $30.5^{\circ} \mathrm{C}$ e $31.5^{\circ} \mathrm{C}$ deram origem a $15 \%$ de machos, e a temperaturas superiores a $31.5^{\circ} \mathrm{C}$, deram origem a cerca de $50 \%$ de machos.

Com base nestes dados, e utilizando a idéia de Woodward e Murray, nós dividimos a região em três subregiões de desova: região A, com temperatura e vegetação apropriadas para uma temperatura de incubação abaixo da temperatura crítica, onde os ninhos forneçam apenas fêmeas; região $\mathrm{B}$, tal que a temperatura de incubacao esteja entre $30.5^{\circ} \mathrm{C}$ e $31.5^{\circ} \mathrm{C}$, de modo que ocorra o nascimento de uma porcentagem $\alpha$ de machos e região $\mathrm{C}$, propícia a temperaturas de incubação elevadas tal que os ninhos forneçam apenas machos.

Crocodilianos em geral são muito territoriais e procuram fazer seus ninhos em um local similar ao que eles nasceram, portanto, supõe-se que os animais nascidos em determinada região procurarão fazer seus ninhos na mesma região onde nasceram. Porém, consideramos um número limitado de locais adequados para construção de ninhos, o que obriga os animais a fazerem seus ninhos em outras regiões. A porcentagem de fêmeas que consegue construir seus ninhos em sua região preferida depende da densidade de fêmeas e do número máximo de ninhos da região. Indícios que esta suposição seja verdadeira já foram observados por Waller [5] em Ibera, que constatou a presença de ninhos em locais mais secos, não utilizados anteriormente, com o aumento da população. Além disto, isto previne uma população totalmente feminina, uma vez que o local predileto para desova são os locais mais frios, com temperaturas próximas a que as fêmeas foram geradas.

Matematicamente, o modelo pode ser descrito como:

- Região A

$$
\begin{aligned}
w a(t) & =\gamma \frac{A N f(\boldsymbol{x a}(t))}{A+N f(\boldsymbol{x a}(t))} \\
x a_{1}(t+1) & =p_{0}(t) w a(t) \\
x a_{i+1}(t+1) & =p_{x i}(t) x a_{i}(t) ; i=1, \ldots, N-1,
\end{aligned}
$$

onde $\boldsymbol{x} \boldsymbol{a}(t)$ é o vetor $\left(x a_{1}(t), \ldots, x a_{N}(t)\right)$ onde $x a_{i}$ representa o número de fêmeas na classe etária $i$ nascidas na região $\mathrm{A}$; wa representa o número de ovos na região A; $N f$ é a função que descreve o número de fêmeas aptas a 
desova; $\gamma$ é o número médio de ovos por ninho; $p_{0}$ é a taxa de sobrevivência dos ovos; $p_{x i}$ é a taxa de sobrevivência das fêmeas da classe etária $i ; N$ é o número de classes etárias e $A$ é o número máximo de ninhos permitidos na região $\mathrm{A}$.

A fração $\frac{A N f(x a(t))}{A+N f(x a(t))}$ representa a porcentagem de fêmeas nascidas na região A que consegue construir seus ninhos na região A. Nós consideramos que as fêmeas nascidas na região A não permitirão que fêmeas oriundas de outras regiões construam ninhos na região A.

- Região B

$$
\begin{aligned}
w b(t) & =\gamma(f a b N f(\boldsymbol{x a}(t))+f b b N f(\boldsymbol{x} \boldsymbol{b}(t))) \\
x b_{1}(t+1) & =(1-\alpha) p_{0}(t) w b(t) \\
x b_{i+1}(t+1) & =p_{y i}(t) x b_{i}(t) ; i=1, \ldots, N-1 \\
y b_{1}(t+1) & =\alpha p_{0}(t) w b(t) \\
y b_{i+1}(t+1) & =p_{y i}(t) y b_{i}(t) ; i=1, \ldots, N-1,
\end{aligned}
$$

onde $\boldsymbol{x} \boldsymbol{b}(t)$ é o vetor $\left(x b_{1}(t), \ldots, x b_{N}(t)\right)$ onde $x b_{i}$ representa o número de fêmeas na classe etária $i$ nascidas na região B; $\boldsymbol{y} \boldsymbol{b}(t)$ é o vetor $\left(y b_{1}(t), \ldots, y b_{N}(t)\right)$ onde $y b_{i}$ representa o número de machos na classe etária $i$ nascidos na região $\mathrm{B} ; w b$ representa o número de ovos na região $\mathrm{B} ; p_{y i}$ é a taxa de sobrevivência dos machos da classe etária $i$ e $B$ é o número máximo de ninhos permitidos na região B. A fração

$$
f a b=\frac{B}{B+N f(\boldsymbol{x a}(t))+N f(\boldsymbol{x} \boldsymbol{b}(t))} \frac{N f(\boldsymbol{x a}(t))}{A+N f(\boldsymbol{x a}(t))}
$$

representa a porcentagem de fêmeas nascidas na região A que não obteve sucesso ao construir seus ninhos na região A e que obteve sucesso construindo ninhos na região B; e a fração

$$
f b b=\frac{B}{B+N f(\boldsymbol{x a}(t))+N f(\boldsymbol{x} \boldsymbol{b}(t))}
$$

representa a porcentagem de fêmeas nascidas na região B que conseguiu construir seus ninhos na região B.

- Região C

$$
\begin{aligned}
w c(t) & =\gamma(f a c N f(\boldsymbol{x a}(t))+f b c N f(\boldsymbol{x b}(t))) \\
y c_{1}(t+1) & =p_{0}(t) w c(t) \\
y c_{i+1}(t+1) & =p_{y i}(t) y c_{i}(t) ; \quad i=1, \ldots, N-1,
\end{aligned}
$$

onde $\boldsymbol{y} \boldsymbol{c}(t)$ é o vetor $\left(y c_{1}(t), \ldots, y c_{N}(t)\right)$ onde $y c_{i}$ representa o número de machos na classe etária $i$ nascidos na região $\mathrm{C}$; $w c$ representa o número de 
ovos na região $\mathrm{C}$ e $C$ é o número máximo de ninhos permitidos na região $\mathrm{C}$. A fração

$$
f a c=\frac{C}{C+N f(\boldsymbol{x a}(t))+N f(\boldsymbol{x} \boldsymbol{b}(t))} \frac{N f(\boldsymbol{x a}(t))+N f(\boldsymbol{x} \boldsymbol{b}(t))}{B+N f(\boldsymbol{x a}(t))+N f(\boldsymbol{x} \boldsymbol{b}(t))} \frac{N f(\boldsymbol{x a}(t))}{A+N f(\boldsymbol{x a}(t))}
$$

representa a porcentagem de fêmeas nascidas na região A que não obteve sucesso ao construir seus ninhos nas regiões A e B e que obteve sucesso construindo ninhos na região C; e a fração

$$
f b c=\frac{C}{C+N f(\boldsymbol{x a}(t))+N f(\boldsymbol{x} \boldsymbol{b}(t))} \frac{N f(\boldsymbol{x a}(t))+N f(\boldsymbol{x} \boldsymbol{b}(t))}{B+N f(\boldsymbol{x a}(t))+N f(\boldsymbol{x} \boldsymbol{b}(t))}
$$

representa a porcentagem de fêmeas nascidas na região $\mathrm{B}$ que não obteve sucesso ao construir ninhos na região B e construiu seus ninhos na região $\mathrm{C}$.

\section{A Dependência sobre o Nível da Água}

Nas equações (2.1)-(2.3), $N f, p_{x i}, p_{y i}$ dependem fortemente do nível da água do pântano. Isto porque diferentes níveis de água e chuva afetam o comportamento dos animais e do ecossistema como um todo, já que o nível da água está relacionado com a quantidade de alimento disponível. Infelizmente, não há qualquer dado estatístico sobre os efeitos do nível da água sobre populações de C. yacare. Para superar esta dificuldade, adaptamos alguns dados sobre o Alligator mississipiensis para o C. yacare. Uma vez que crocodilianos em geral são muito semelhantes, as aproximações mostradas a seguir refletem o modo com que o nível da água afeta a taxa de mortalidade, porém seus valores são fictícios. Isto certamente compromete a confiabilidade dos resultados numéricos; mas não afeta a dinâmica do sistema, que é nosso objeto de estudo. ${ }^{1}$

\section{O número de fêmeas ativas e o nível da água}

O nível da água afeta a corte dos animais, consequentemente influindo no número de fêmeas atuantes. Uma quantidade grande de chuvas (e portanto quanto maior o nível da água no pântano) beneficia a corte, aumentando a porcentagem de fêmeas aptas a gerar filhotes. Nós utilizamos basicamente a mesma função (Figura 1) utilizada em [3]. A função $N f(\boldsymbol{x})\left(\boldsymbol{x}=\left(x_{1}, \ldots, x_{N}\right)\right)$ citada nas equações (2.1)(2.3) é definida por

$$
N f(\boldsymbol{x})=\beta(\bar{w} d) \sum_{i=6}^{N} x_{i}
$$

onde $\beta(w d)$ é a função descrita pela figura 1, que representa a porcentagem de fêmeas ativas em função do nível da água.

\footnotetext{
${ }^{1}$ Nas figuras 1-6, um nível da água $w d=0$ significa um nível de água normal e um nível de água $w d=-60$ significa uma forte seca.
} 


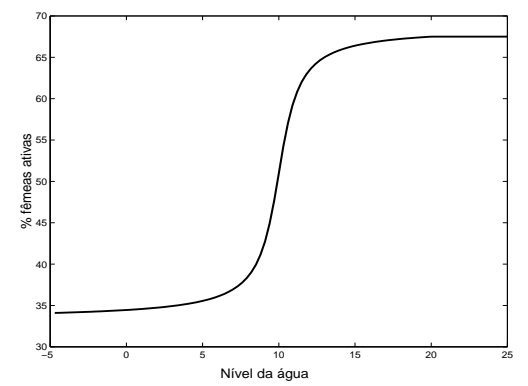

Figura 1: Porcentagem de fêmeas reprodutoras em função do nível da água

\section{Taxa de sobrevivência dos ovos}

O nível da água afeta a sobrevivência dos ovos de duas maneiras (Figura 2). Primeiro, os ovos não são resistentes a enchentes, e perdem-se todos os ovos em caso de grandes quantidades de chuva. Segundo, o nível da água afeta a abundância de alimento, o que afeta a predação dos ovos. As funções utilizadas foram obtidas de dados reais obtidos em pesquisa de campo [3]. Assim, a taxa de sobrevivência para os ovos pode ser escrita como

$$
p_{0}(t)=\operatorname{sflood}(\boldsymbol{w d}(t)) \operatorname{spred}(\boldsymbol{w d}(t)),
$$

onde sflood é a taxa de sobrevivência a enchente e spred é a taxa de sobrevivência a predação .
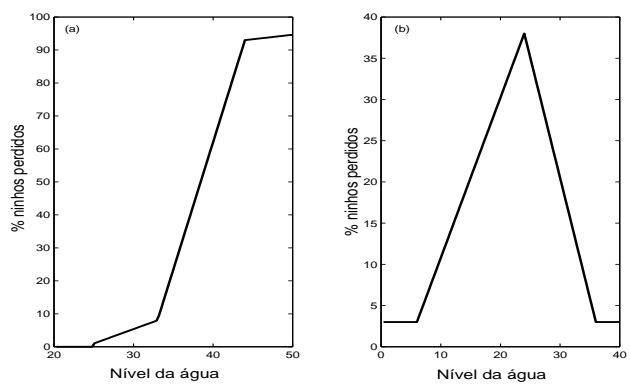

Figura 2: (a)Taxa de mortalidade referente a inundação dos ninhos e (b) taxa de mortalidade referente a predação dos ninhos em função do nível da água.

\section{Taxa de sobrevivência da população}

Assim como ocorre com os ovos, o nível da água influi na taxa de predação dos jovens e recém-nascidos. Além destes fatores, níveis de água muito baixos podem 
provocar morte por desidratação. Nós utilizamos as mesmas relações utilizadas em [3], que são mostradas na figura 3.
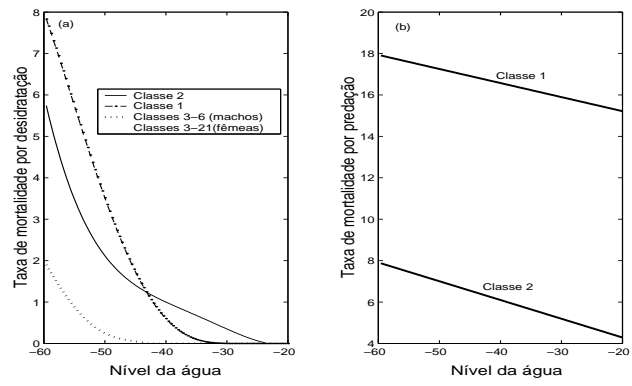

Figura 3: (a)Taxa de mortalidade referente a desidratação e (b) taxa de mortalidade referente a predação em função do nível da água.

\section{Canibalismo}

Existem evidências de que ocorra canibalismo entre crocodilianos. É natural considerar que as taxas de canibalismo dependem não somente da densidade, mas também da abundância de alimento, que está relacionada com o nível da água. Assim a taxa de mortalidade por canibalismo é o produto de uma componente dependente da densidade (figura 4(a)) e um fator multiplicativo dependente do nível da água (figura 4(b)). Além disso, os animais mais jovens são mais afetados pelo canibalismo.
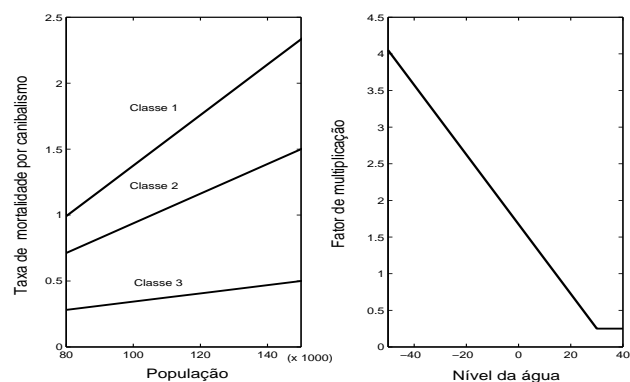

Figura 4: (a)Taxa de mortalidade referente a canibalismo. (b)Fator multiplicativo da taxa de canibalismo em função do nível da água.

\section{Morte natural}

Além das mortes por causas específicas, é considerada também a mortalidade por causas naturais (doenças e acidentes), dependente somente da idade dos indivíduos 


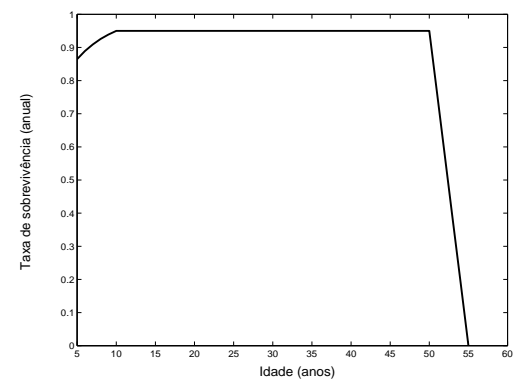

Figura 5: Mortalidade por causas naturais em função da idade dos indivíduos

(Figura 5). Assim, a taxa de sobrevivência para a população na classe $i$ de sexo $s$ no tempo $t$ pode ser escrita como

$$
p_{s i}=p(i) \prod_{k=1}^{12} \operatorname{sdesic}\left(s, i, w d_{k}\right) \operatorname{spred}\left(i, w d_{k}\right) \operatorname{scann}\left(i, w d_{k}\right) \text {, }
$$

onde $p(i)$ é a taxa de sobrevivência anual a causas naturais (função descrita na figura 5$), w d_{k}$ é a média mensal do nível da água, sdesic é a probabilidade mensal de sobrevivência a desidratação, spred é a probabilidade mensal de sobrevivência a predação e scann é a probabilidade mensal de sobrevivência ao canibalismo.

\section{Estratégias de Desenvolvimento}

\section{Coleta de ovos}

Coleta de ovos está entre os possíveis usos comerciais da população. Trata-se de recolher um certo número de ovos de seus ninhos, incubá-los em laboratório e cuidar dos filhotes até que eles alcancem um certo tamanho. Após, uma porcentagem destes animais é devolvida para o meio ambiente, e os restantes são sacrificados para a retirada da pele. As vantagens são que os ovos são salvos das altas taxas de predação e das enchentes e os filhotes são em grande parte salvos das altas taxas de mortalidade encontradas no meio selvagem (predação, canibalismo, desidratação e morte por congelamento). Alem disso, se bem alimentados, estes animais vem a crescer mais, o que leva a uma maior taxa de sobrevivência no meio selvagem. Assim, se os animais libertados forem fêmeas (o que pode ser controlado, já que o sexo dos animais é determinado pela temperatura de incubação dos ovos) um porcentual pequeno de reposição é suficiente para a sobrevivência da espécie. As desvantagens são que as regras quanto a alimentação e acomodação dos animais devem ser muito estudadas, já que uma alimentação diferente da normal pode vir a diminuir a taxa de sobrevivência dos animais.

Apesar da necessidade de estudos aprofundados em relação aos aspectos biológicos dos animais criados em cativeiro, nosso modelo incluiu a possibilidade da coleta 
de ovos, com algumas suposições. Supomos que os animais em cativeiro possuem uma taxa de sobrevivência diferenciada e que os animais libertos terão as mesmas chances de sobrevivência que um animal criado no meio. Quando as fêmeas são libertadas, supomos que estas competirão para colocarem seus ninhos na região A com as fêmeas da região A, por duas razões: primeiro, esta é a melhor região, segundo, dado que estes animais são fêmeas, foram incubadas a temperaturas abaixo da temperatura crítica, e vão procurar por um local com temperatura semelhante para construir seus ninhos, que por hipótese, está na região A. Assim as expressões para $w a, w b$ e $w c$ tornam-se

$$
\begin{aligned}
w a(t) & =\gamma \frac{A N f(\boldsymbol{x a}(t)+\boldsymbol{x l}(t))}{A+N f(\boldsymbol{x a}(t)+\boldsymbol{x l}(t))} \\
w b(t) & =\gamma \frac{A N f(\boldsymbol{x a}(t)+\boldsymbol{x l}(t))}{A+N f(\boldsymbol{x a}(t)+\boldsymbol{x l}(t))} \frac{B}{B+N f \operatorname{cotal}(t)} N f \operatorname{total}(t) \\
w c(t) & =\gamma \frac{N f \operatorname{total}(t)}{B+N f \operatorname{total}(t)} \frac{C}{C+N f \operatorname{total}(t)}\left(N f(\boldsymbol{x b}(t))+\frac{A(N f(\boldsymbol{x a}(t)+\boldsymbol{x l}(t)))^{2}}{A+N f(\boldsymbol{x a}(t)+\boldsymbol{x l}(t))}\right),
\end{aligned}
$$

onde $x l_{i}$ e $y l_{i}$ representam a população de fêmeas e machos devolvidas ao meio ambiente na classe $i$ e $N f \operatorname{total}(t)=N f(\boldsymbol{x} \boldsymbol{a}(t)+\boldsymbol{x} \boldsymbol{b}(t)+\boldsymbol{x l}(t))$ representa o número total de fêmeas em desova no tempo $t$.

\section{Caça}

Um dos maiores objetivos deste trabalho é analisar os efeitos da caça sobre a população. Por esta razão, incluimos a opção para a caça no programa de simulação, de acordo com um tamanho mínimo exigido pré-definido pelo usuário. Para isto, utilizamos a relação entre tamanho SVL (snout-vent lenght) e idade obtida por Rebélo[4], dada por (veja figura 6)

$$
\begin{aligned}
& a_{f}(s)=5.663 \ln \left(\frac{819}{1036-10 s}\right) \\
& a_{m}(s)=7.013 \ln \left(\frac{1041}{1261-10 s}\right)
\end{aligned}
$$

onde $a_{f}(s)\left(a_{m}(s)\right)$ representa a idade da fêmea (macho) de tamanho $s$. Assim, se um número $H$ de animais maiores que um tamanho $s$ são caçados, uma mortalidade $h(i, H)=\frac{H}{\text { Total }}$ é aplicada à cada população $x a_{i}(t), x b_{i}(t), x l_{i}(t)$ para $i>a_{f}(s)$ e à cada população $y b_{i}(t), y c_{i}(t), y l_{i}(t)$ para $i>a_{m}(s)$. Total representa o número total de animais sujeitos a caça, ou seja,

$$
\text { Total }=\sum_{k=a_{f}}^{N} x a(t)+x b(t)+x l(t)+\sum_{k=a_{m}}^{N} y b(t)+y c(t)+y l(t) .
$$



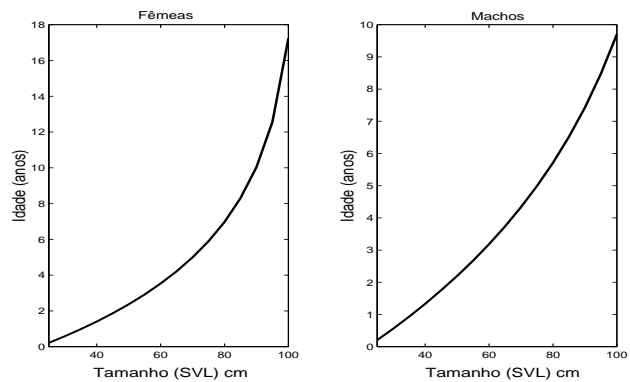

Figura 6: Relação entre tamanho e idade para (a)fêmeas e (b)machos.

\section{Resultados}

O modelo apresentado aqui é o primeiro esboço que obtivemos. Esperamos que o programa de simulação forneça resultados úteis para a análise dos efeitos da caça e coleta de ovos. Na Figura 7 temos uma simulação feita com $w d=0$ e os dados mostrados nas figuras 1-6 para a taxa de sobrevivência, para três casos: sem nenhuma estratégia de desenvolvimento, com caça de $10 \%$ sobre os animais maiores que $90 \mathrm{~cm}$, que reduz sensivelmente a capacidade de carga da população, e com caça e coleta de ovos, que mostra uma capacidade de carga superior. Ainda que os valores não sejam realísticos, este resultado já pode ser considerado útil em relação ao comportamento dinâmico: Waller [5] observou um crescimento exponencial no último senso realizado em Ibera, após um periodo de 10 anos sem caça, o mesmo comportamento exibido na figura 7.

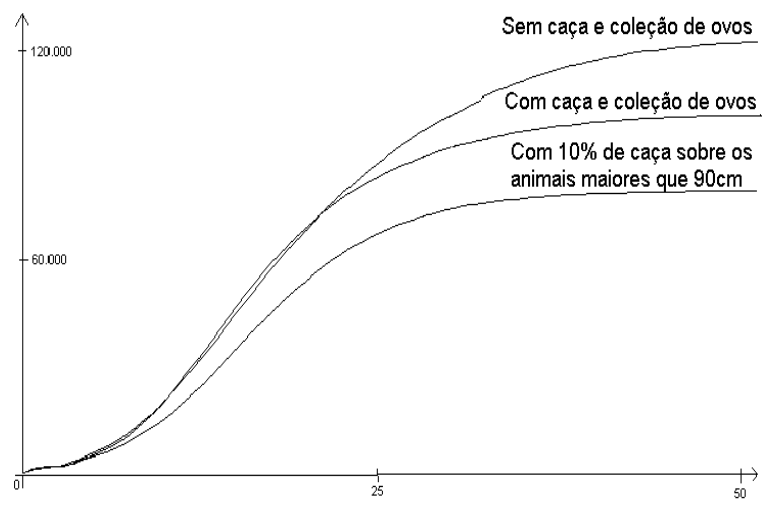

Figura 7: Resultados preliminares obtidos pelo programa de simulação.

Abstract. A nonlinear age structured model was constructed for the Ibera $C$. yacare population. We consider the placement of the nests (related to the tem- 
perature in the nest) as the determining factor for the sex of the hatchlings. The dynamical behaviour and the effects of management strategies as harvest and eggs collection are studied through numerical simulation.

\section{Referências}

[1] G.E.D. Blomberg, B.C.St. Pierre, K.D. Smith, S.M. Caddell e S.R. Pett, Simulated population dynamics of crocodiles in the Okavango river, Botswana. Crocodiles, em "5th Working Meeting of the Crocodile Specialist Group of the SSC/IUCN", Gainesville, Florida, 1980, UICN, Gland, Suiza, 1982.

[2] Z. Campos, Effects of habitat on survival of eggs and Sex ratio of hatchlings of Caiman crocodilus yacare in the Pantanal, Brazil, Journal of Herpetology 27 (1993), 127-132.

[3] J.D. Nichols, L. Viehman, R.H. Chabreck e B. Fenderson, Simulation of a commercially harvested alligator population in Louisiana, Louisiana State Univ. Agric. Exper. Sta. Bull. 691, 1976.

[4] G.H. Rebélo, G.A.N. Borges, C. Yamashita e A.G. Arruda Filho, Growth, Sex ratio population structure and hunting mortality of Caiman yacare in the Pantanal, Brazil, Vida Silvestre Neotropical 6 (1997), 29-36.

[5] T. Waller, Situación del genero Caiman en Argentina. The Sustainable Management of Wetland Resources in Mercorsur-II Meeting (em preparação).

[6] D.E. Woodward e J.D. Murray, On the effect of temperature-dependent sex determination on sex ratio and survivorship in crocodilians, Proc. R. Soc. Lond. B 252 (1993), 149-155. 